\title{
Enfoque para la clasificación de vegetación polinífera usando imágenes multiespectrales y redes neuronales
}

\author{
Juan Jose Negron-Granados, Ricardo Legarda-Sáenz, Víctor Uc-Cetina \\ Universidad Autónoma de Yucatán, Facultad de Matemáticas, \\ México \\ a08001373@alumnos.uady.mx, \{rlegarda,uccetina\}@correo.uady.mx
}

\begin{abstract}
Resumen. La apicultura es una actividad pecuaria muy importante para México. Yucatán es uno de las entidades federativas que más aporta en producción y exportación de miel. Sin embargo, la apicultura se ve amenazada por una deficiente organización de los apicultores, mala ubicación de los apiarios y reducción de la vegetación, lo que afecta a la flora polinífera, principal fuente de alimento de las diferentes especies de abejas. Este trabajo hace la propuesta de utilizar información espectral para identificar las diferentes plantas productoras de polen utilizando visión remota, procesamiento de imágenes y redes neuronales artificiales. En este trabajo se sientan las bases para realizar un software que contribuya a los apicultores en la cuantificación de la flora polinífera que existan alrededor de sus apiarios y les sirva como herramienta para prevenirse ante hambrunas, optimización en la ubicación estratégica de colonias de abeja y mejorar una de las actividades pecuarias más importantes de México.
\end{abstract}

Palabras clave: Índices de vegetación, redes neuronales, imágenes multiespectrales.

\section{Approach for the Classification of Polinífera Vegetation Using Multispectral Images and Neural Networks}

\begin{abstract}
The apiculture is a very important livestock activity for Mexico. Yucatan is one of the states that contributes most in the production and export of honey. However, the apiculture is threatened by a poor organization of beekeepers, poor location of the apiaries and reduction of vegetation, which affects the polliniferae flora, main source of food for different species of bees. This work makes the proposal to use spectral information to identify the different pollen producing plants using remote viewing, image processing and artificial neural networks. In this work, the foundations are laid for making software that contributes to beekeepers in the quantification of the polliniferae flora that exist around their apiaries and serves them as a tool to prevent themselves from famines,
\end{abstract}


optimization in the strategic location of bee colonies and improve one of the most important livestock activities in Mexico.

Keywords: Vegetation indices, neural networks, multispectral images

\section{Introducción}

La península de Yucatán es, por tradición, una importante región productora de miel a nivel mundial, ya que $95 \%$ de su producción se destina al mercado internacional [12]. La apicultura ha sufrido un grave deterioro en la región. Ha disminuido la posibilidad de recursos néctar-polinívoros y, por tanto, la alimentación de las abejas, con la consiguiente baja en la producción. Por lo que existe la necesidad de mejorar los sistemas de comercialización y de diversificación de la actividad y actualizar las técnicas de producción y administración del proceso productivo por parte de los apicultores para obtener la calidad de la miel requerida por el mercado [12].

Una manera de mejorar la producción y la calidad de la miel es caracterizando la vegetación que se encuentra alrededor de una colonia de abejas. La dieta de las abejas es muy específica (Tabla 1), por lo que se espera que $5 \mathrm{~km}$ a la redonda de un apiario se encuentre una abundante vegetación adecuada para evitar los problemas de producción de miel.

Tabla 1. Especies de plantas más importantes para la producción de miel en Yucatán.

\begin{tabular}{|l|l|}
\hline Nombre común & Nombre científico \\
\hline Ts'its'ilche' & Gymnopodium floribundum \\
\hline Taj & Viguiera dentata \\
\hline Ja'abin & Piscidia piscipula \\
\hline Box katsim & Acacia gaumeri \\
\hline Sac katsim & Mimosa bahamensis \\
\hline Tsalam & Lysiloma latisiliquum \\
\hline X-tabentún & Turbina corymbosa \\
\hline Kitimché & Caesalpinia gaumeri \\
\hline Enredaderas & Varias especies de la familia Convolvulaceae \\
\hline
\end{tabular}

La clasificación de plantas en salidas de campo es un proceso complicado y nada eficiente. Es común que para la evaluación y clasificación de la vegetación suelen usarse imágenes satelitales por su alto contenido de información. Sin embargo, la distancia de los sensores evita tener imágenes de buena resolución, pues en una imagen multiespectral satelital un pixel puede representar un área de 30 $m^{2}$. Obtener imágenes aéreas con un dron mejora resolución espacial. La cámara Sequoia Parrot permite obtener imágenes con una con una relación de 3,7 y 18,6 centímetros por pixel para vuelos de 30 y 150 metros de altura respectivamente. 
Enfoque para la clasificación de vegetación polinífera usando imágenes multiespectrales ...

Con la información de los índices de vegetación se pueden calcular los Valores medios, desvíos estándares, máximos y mínimos valores entre índices espectrales que se utilizan como el vector de características de la red neuronal.

En este artículo se ha presentado en la sección de introducción la descripción del problema que se tiene con la flora polinífera, y la importancia y los beneficios que conlleva la clasificación de dicha flora. A continuación, en la sección del estado del arte, se describen los trabajos relacionados que se utilizó para explorar las diferentes técnicas empleadas para resolver problemas similares y justificar la metodología empleada. Posteriormente, se describe la metodología propuesta y las herramientas a utilizar para cumplir nuestro objetivo de clasificar diferentes plantas apícolas. Luego se presentan los resultados preliminares que dan soporte de factibilidad a la metodología propuesta.

\section{Trabajos en el área de clasificación de imágenes espectrales}

En la literatura consultada podemos encontrar trabajos similares, en los cuales se utilizan algoritmos de aprendizaje automático para el reconocimiento de superficies usando imágenes satelitales. A continuación se mencionan los trabajos enfocados a resolver problemas de clasificación de coberturas usando métodos de aprendizaje profundo (Deep Learning); estos trabajos se caracterizan por hacer uso de imágenes hiperespectrales. Las imágenes hiperespectrales están compuestas de imágenes contiguas en todo el espectro electromagnético, pudiendo llegar a ser un conjunto de más de 100 imágenes.

Los trabajos más recientes acerca de la clasificación general de la cobertura terrestre utilizan técnicas de aprendizaje profundo y se encuentran en las investigaciones de Chen y Lin, del Instituto de Tecnología Harbin, China. En el año 2013 Lin et al. hacen la propuesta de utilizar autoencoders para la clasificación de imágenes hiperespectrales [14]. En el 2014, Chen et al. reportaron sus resultados de aplicar los autoencoders [5] para el reconocimiento de diferentes regiones terrestres, logrando identificar con mucho éxito: suelo desnudo, prados, ladrillos, sombras, grava, agua, diferentes tipos de pantano, maleza y pinos. Un año después, Chen et al. realizaron el mismo experimento, cambiando el método autoencoder por una red de creencia profunda (DBN por sus siglas en inglés, Deep Belief Network) [6]. Los resultados fueron bastante buenos, la red pudo identificar las mismas regiones que detectó la técnica de autoencoder, sin embargo la arquitectura del autoencoder supera ligeramente a DBN. En promedio el autoencoder tuvo una efectividad del $96 \%$ y DBN del $93 \%$.

También es posible encontrar trabajos que empleen técnicas de Redes Neuronales Convolucionales (CNN, por sus siglas en inglés), por ser métodos efectivos para el procesamiento de imágenes y la extracción de características. En las pruebas de Makantasis [17] y Maggiori [16] obtienen resultados de efectividad del $99 \%$.

Los trabajos anteriores describen la metodología para clasificar las coberturas utilizando métodos de aprendizaje profundo utilizando imágenes hiperespectra- 
les, las cuales resultaron ser altamente eficientes. Sin embargo, en la literatura hay metodologías que utilizan imágenes multiespectrales, este tipo de imágenes consta de un conjunto entre 4 y 20 imágenes espectrales, dependiendo del satélite o sensor que se utilice. Para el procesamiento de imágenes multiespectrales en vegetación se suelen usar 4 bandas del espectro de la luz: verde $(530-570 \mathrm{~nm})$, rojo $(640-680 \mathrm{~nm})$, borde rojo $(730-740 \mathrm{~nm})$ e infrarrojo cercano $(770-810 \mathrm{~nm})$. A continuación se mencionan algunos de los trabajos citados que utilizan las bandas espectrales mencionadas para la identificación de las diferentes coberturas; además cabe mencionar que se utiliza una Red Neuronal Artificial como método de clasificación y sensores satelitales para la obtención de la información espectral.

El uso de redes neuronales artificiales en la clasificación de datos de sensores remotos ha crecido en los últimos años [15]. En la literatura citada se han usado para identificar diferentes coberturas terrestres, como agua, asfalto, suelo desnudo, vegetación $[3,26]$.

Entre las aplicaciones más importantes se encuentra el análisis multitemporal [11] que consiste en la clasificación de las coberturas pero analizando las variaciones espectrales a través del tiempo para determinar los cambios en las características biofísicas de las regiones, determinar el avance de la restauración de zonas ecológicas [23] o el monitoreo de parques nacionales [25]. Del mismo modo, la técnica de combinar imágenes multiespectrales con redes neuronales ha llevado al análisis de cultivos, por ejemplo el de caña de azúcar [24] o el análisis de las condiciones del suelo, como el nivel de salinidad en la tierra [19].

Debido a que varias coberturas terrestres pueden presentar respuestas espectrales similares, se han realizado variantes que complementan la información de las imágenes con información cartográfica [18] como modelos digitales de elevación (MDE), mapas temáticos de la región o datos de precipitación atmosférica [1], mejorando la efectividad aproximadamente en un $10 \%$.

En los trabajos presentados anteriormente se describen las ventajas de utilizar las imágenes multiespectrales y las redes neuronales para la clasificación de diferentes coberturas del suelo, sin embargo, una de las limitaciones de utilizar imágenes satelitales es el costo de la adquisición y procesamiento de las imágenes, pues una imagen puede tener una dimensión mayor a 27000 pixeles $^{2}$ con una resolución de 11 bits (QuickBird) por cada una de las bandas que opera el sensor en el satélite. Otra limitante que es común observar es la resolución del píxel, en el satélite QuickBird se tiene una resolución de $61 \mathrm{~cm}$ de resolución, esto quiere decir que un pixel representa un área de $0,36 \mathrm{~m}^{2}$; esta resolución puede ser útil cuando se trata de clasificar superficies extensas, sin embargo para detectar plantas específicas requiere una resolución más precisa.

Adicionalmente, en los trabajos presentados se identifica el proceso general para la clasificación de coberturas usando imágenes espectrales: adquisición de las imágenes, un preprocesamiento en la imagen, como el cálculo de los índices de vegetación o las correcciones y calibración de las imágenes satelitales, finalmente se hace el uso de técnicas de algoritmos de aprendizaje máquina para la clasificación. 
Enfoque para la clasificación de vegetación polinífera usando imágenes multiespectrales ...

\section{Materiales y métodos}

A diferencia de la literatura citada, este trabajo propone utilizar utilizando un dron y la cámara multiespectral Sequoia Parrot para muestrear las áreas de interés para la recolección de las muestras de la flora apícola. Esto debido a que mejora la resolución espacial de las imágenes y se evita influencias de gases en la atmósferas y nubes. Posteriormente se propone calcular índices de vegetación de las imágenes muestreadas; estos índices son el índice de vegetación de diferencia normalizada, el índice de vegetación verde normalizada y el índice de clorofila. A partir de los índices de vegetación calculados se construirá el vector de características a partir de datos estadísticos: promedio, moda, mínimo y máximo de cada uno de los índices. En total, un vector de 12 características para cada una de las imágenes. Luego, se generará una base de datos etiquetada con los vectores de características calculados. A continuación se diseña, programa y entrena una red neuronal completamentamente conectada para la clasificación de la vegetación utilizando la base de datos etiquetada. Una vez entrenada la red se probará el clasificador y finalmente, se analizarán los resultados. En las siguientes subsecciones se describen los materiales necesarios para llevar a cabo la metodología propuesta.

\subsection{Determinación de los datos espectrales}

Las imágenes a utilizar corresponden al sensor multiespectral Sequoia Parrot (Figura 1). Este sensor está compuesto por dos módulos: el cuerpo (Body) y el sensor de luz (Sunshine sensor). El cuerpo está integrado por cuatro cámaras espectrales de 1.2 Mpx, una cámara RGB de 16 Mpx, WiFi, IMU y magnetómetro. El sensor de luz cuenta con 4 sensores espectrales con los mismos filtros que el cuerpo, GPS, IMU y magnetómetro. La información de las diferentes frecuencias de la luz que se pueden obtener son las bandas: verde (530-570 $\mathrm{nm}$ ), rojo $(640-680 \mathrm{~nm})$, borde rojo $(730-740 \mathrm{~nm})$ e infrarrojo cercano $(770-810 \mathrm{~nm})$; además de tomar fotos a color (Imágenes RGB). Las imágenes de las 4 bandas espectrales tienen formato RAW de 10 bits en un archivo TIFF. La imagen RGB se guarda en formato JPG. En total son 5 imágenes por cada foto.

Con la información de las imágenes multiespectrales es posible calcular índices espectrales que constituyen una herramienta útil para evaluar áreas extensas [2]. Recientes avances en el aprendizaje profundo han hecho que hoy en día sea posible utilizar estos índices para resolver problemas relacionados con la identificación de elementos en una imagen, con métodos coherentes, precisos y fiables [9].

\subsection{Análisis de datos}

Existen combinaciones de las imágenes espectrales obtenidas para obtener información relevante de la vegetación, a esto se le conoce como índices de vegetación. Estos índices permiten estimar variables biofísicas de la vegetación a partir de la luz que las plantas emiten o reflejan, minimizando la influencia de 

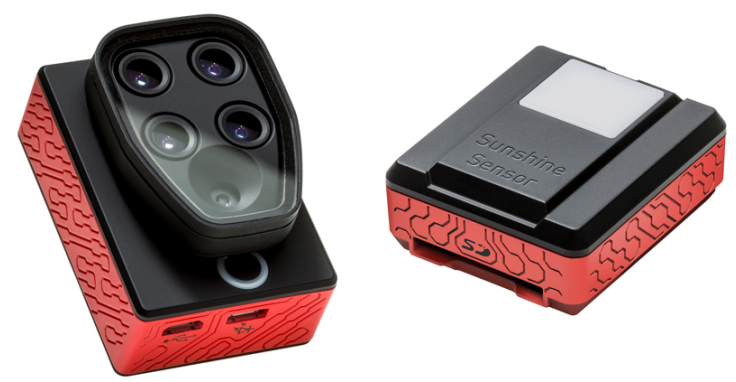

Fig. 1. Cuerpo del sensor (Izquierda). Sensor de luz (Derecha).

perturbaciones como las debidas al suelo y a las condiciones atmosféricas [10,21].

La selección de bandas multiespectrales depende del índice que se desee calcular [10]; sin embargo, es frecuente el uso de las bandas espectrales del rojo (del campo visible) y el infrarrojo cercano, puesto que las plantas reflejan fuertemente la banda de luz infrarroja cercana debido a una capa gruesa en la superficie inferior de la hoja (Figura 2). A continuación se describen los índices de vegetación a utilizar para la extracción de características.

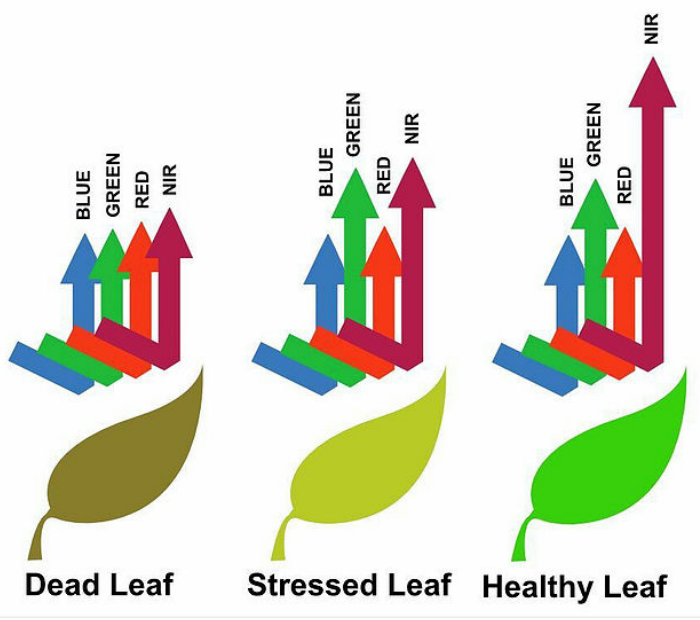

Fig. 2. El pigmento de las hojas de las plantas, la clorofila, absorbe con fuerza la luz visible para su uso en la fotosíntesis. La estructura celular de las hojas, por otro lado, refleja intensamente luz infrarroja cercana [20].

Índice de Vegetación Normalizado Rouse propuso el Índice de Vegetación de Diferencia Normalizada (Normalized Difference Vegetation Index, NDVI) en 
1974 y hasta la fecha es uno de los más usado [22]. Un aspecto interesante de este índice frente al cociente simple es que toma valores entre -1 y 1 , lo cual facilita notablemente su interpretación [13]. El NDVI es la diferencia normalizada de las bandas infrarroja (NIR) y roja (R) (Ecuacion 1$)$ :

$$
N D V I=\frac{N I R-R}{N I R+R} .
$$

Este índice es uno de los más usados, pues está fuertemente relacionado con la salud de las plantas. Una planta sana tiene las paredes de las células en las hojas llenas de agua; por lo que reflejan fuertemente la luz infrarroja. Por otro lado, una planta que sufre de estrés por plagas o sequías, reflejan débilmente la banda de infrarrojo, pues sus hojas no cuentan con suficiente agua.

Índice de Vegetación de Diferencia Normalizada Verde El Índice de Vegetación de Diferencia Normalizada Verde (Green Normalized Vegetation Index, GNVI) es un índice de la actividad fotosintética, y es uno de los índices de vegetación más comúnmente utilizados para determinar la absorción de agua y nitrógeno en el follaje del cultivo [7]. Es similar al NDVI, sin embargo se sustituye la banda roja del espectro visible por la banda verde del espectro visible (V) (Ecuación 2). Siendo uno de los índices de vegetación más comúnmente utilizados para determinar la absorción de agua y nitrógeno en el follaje del cultivo [4]:

$$
G N V I=\frac{N I R-V}{N I R+V}
$$

Índice de Vegetación de Clorofila El índice de vegetación de clorofila (Chlorophyll vegetation index, CVI) es un estimador de clorofila [27]. Se calcula usando las bandas del infrarrojo cercano, roja y verde (Ecuación 3):

$$
C V I=N I R \cdot \frac{R}{G^{2}}
$$

\subsection{Red neuronal artificial}

Las redes neuronales artificiales $(\mathrm{RN})$ son algoritmos matemáticos basados en el funcionamiento del cerebro. Es un método de aprendizaje es supervisado, es decir que tiene una fase de entrenamiento. Existe un gran número de arquitecturas de RN; la propuesta para este trabajo es una perceptrón multicapa con dos capas ocultas completamente conectadas (Figura 3), debido a que la más empleada en percepción remota [8] por ser más eficiente que otros métodos para la clasificación de coberturas terrestres [15].

Para el entrenamiento de la red se propone extraer información estadística de los índices de vegetación para generar las entradas a la red neuronal, a estas entradas se le denomina como vector de características. Los parámetros estadísticos propuestos son los valores de media, moda, máximo y mínimo de cada índice utilizado. En total el vector de características esta compuesto de 12 parámetros: media, moda, máximo y mínimo de NDVI, GNDVI y CIR. 


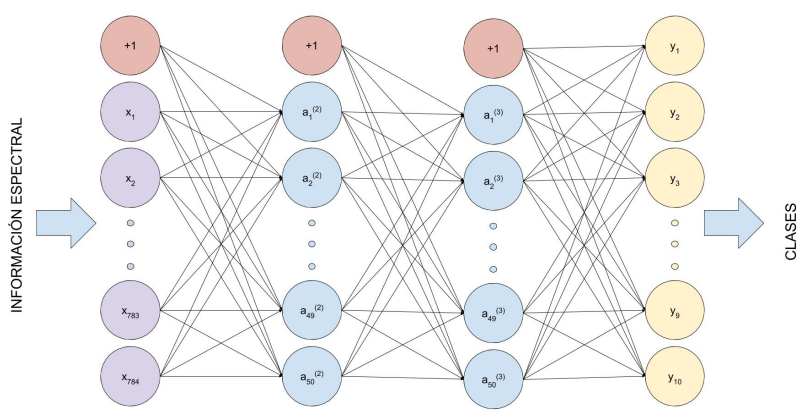

Fig. 3. Estructura de la red neuronal.

\section{Análisis espectral}

Como se ha mencionado en la sección 2, la literatura citada utiliza imágenes satelitales para la clasificación de diferentes texturas, sin embargo, dentro la textura vegetación no se ha reportado la clasificación de plantas específicas, posiblemente debido a la resolución especial de los sensores en los satélites. En la sección 3, se explican las razones de utilizar imágenes multiespectrales aéreas en los drones para obtener una mejor resolución espacial, las diferencias espectrales de cada planta nos sirve como un indicador único de información para cada tipo de planta, a esto se le conoce como firma espectral. En este apartado se hace una comparación de diferentes datos espectrales entre 2 plantas cítricas, limón y naranja (Figura 4), para marcar la diferencia espectral que existe en la diversidad de la flora, lo que evitará datos sesgados en el entrenamiento de la red neuronal y por lo tanto, mejor eficiencia en el clasificador.

Se calcularon los histogramas de las diferentes bandas espectrales (Figura 5), en donde se pudo observar que existen dos montículos en la frecuencia de algunos histogramas, estando la más notoria en la banda roja. Sin embargo, también es posible observar en la banda del borde rojo pero con mayor diferencia en número de la frecuencia. En las bandas infrarrojo y verde, solo es notoria las frecuencias que están relacionadas con el árbol de limón. Luego se calcularon los índices de vegetación: NDVI, GNVI y CVI para analizar los parámetros biofísicos de los árboles (Figura 6). De las imágenes de los índices calculados podemos decir que el árbol de limón tiene mayor densidad de biomasa y mayor concentración de nitrógeno, agua y clorofila en sus hojas que el árbol de naranja. Por lo tanto, ambos tienen información única que permitirá construir un clasificador eficiente.

\section{Conclusiones}

En este trabajo se propone utilizar una cámara multiespectral colocada en un dron para la captura de imágenes. Al usar imágenes dentro de la atmósfera terrestre eliminamos la interferencia que pueden tener los gases, la atmósfera o 


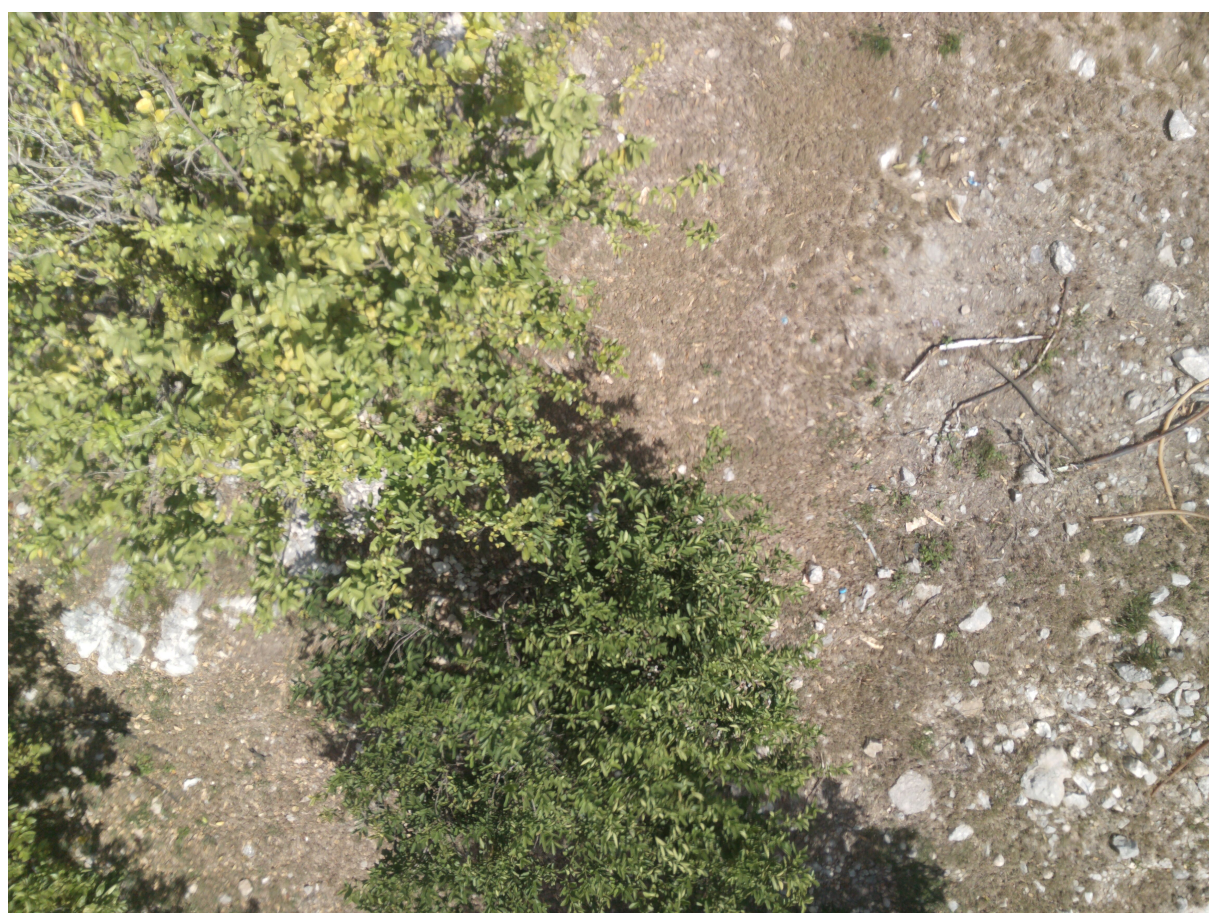

Fig. 4. En la parte superior izquierda de la imagen se encuentra un árbol de naranja, el árbol inferior es de limón.

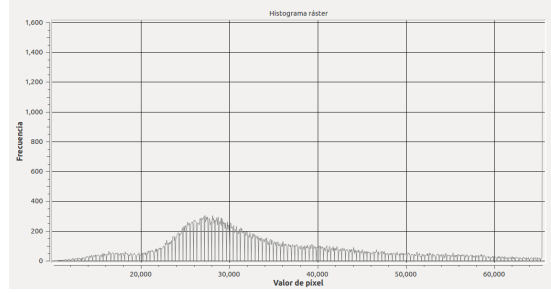

(a) Infrarrojo

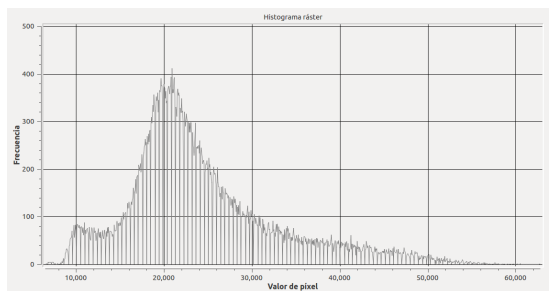

(c) Borde rojo

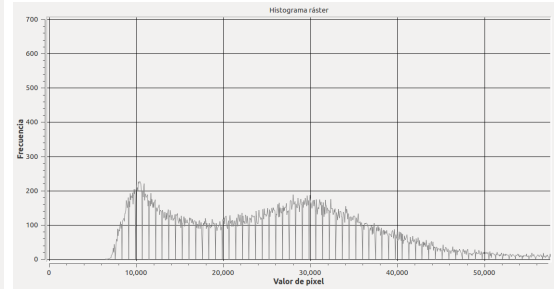

(b) Rojo

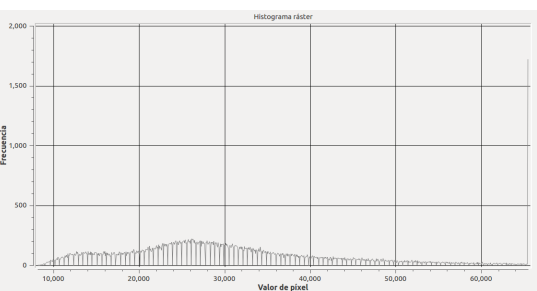

(d) Verde

Fig. 5. Histogramas para las diferentes bandas espectrales. 


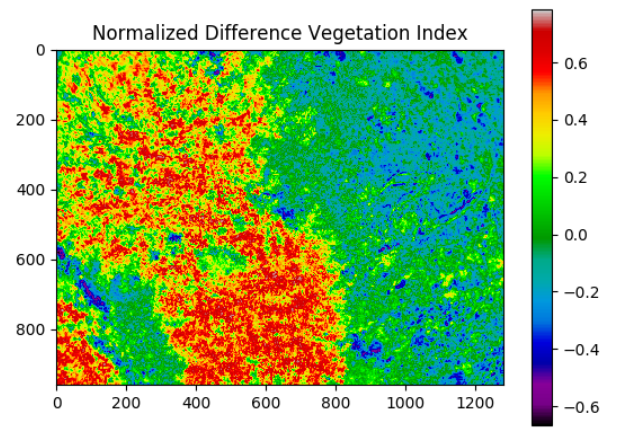

(a) NDVI

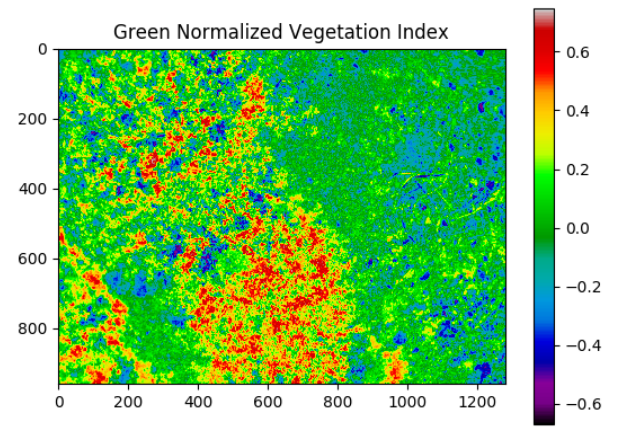

(b) GNVI

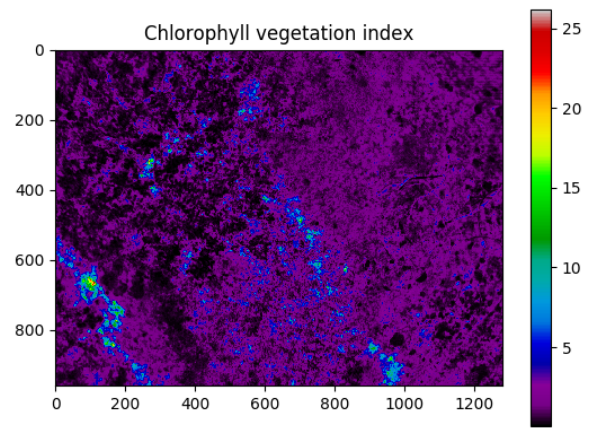

(c) CVI

Fig. 6. Imágenes de los diferentes índices de vegetación. 
las nubes en las imágenes espectrales. La corta distancia permite tener mejor resolución de las imágenes y por lo tanto obtener información más detallada de la cobertura terrestre. Utilizar un vector de descriptores minimiza el tiempo del entrenamiento. El uso de técnicas de aprendizaje máquina, en este caso redes neuronales, y procesamiento de imágenes mejora el análisis de la información espectral. Este trabajo contribuye en la innovación de los procesos en las actividades propias de la apicultura. Mejoraría la organización de los productores. Lograr una distribución óptima de las colonias.

\section{Trabajos futuros}

En el presente trabajo se propone la metodología de implementar una red neural para la clasificación de plantas apícolas específicas; se ha demostrado la factibilidad de usar redes neuronales e imágenes multiespectrales a baja altura para la clasificación; así como también se fundamenta el uso de los índices de vegetación como principales descriptores de la vegetación. Esta metodología podría ser capaz de aportar datos relevantes para impulsar la apicultura endémica de la península de Yucatán, al poder cuantificar de una manera precisa la densidad de los recursos de la flora alrededor de una colmena, incluso ser capaz de identificar el estado de salud de plantas; lo que permitiría optimizar las épocas de cosechas y crianza de la crianza (la crianza de abejas es una actividad en la que el apicultor proporciona alimento a las colmenas debido a la escasez de recursos) de abejas. Sin embargo basado en otros trabajos, sería interesante observar si la factibilidad puede mejorar utilizando arquitecturas de redes artificiales más recientes, como las redes convolucionales profundas o técnicas de deep learning.

En trabajos futuros se puede complementar la información del sensor espectral utilizado información espectral de las cámaras en los satélites; y mejorar o actualizar la información de la floración de vegetación polinífera. De igual manera se podría hacer un análisis de observar como los plaguicidas afectan el comportamiento de las plantas, realizar un estudio de la disminución de las areas acuicolas en busca de mejorar la distribución de los apiarios.

\section{Referencias}

1. Bunrit, S., Chanklan, R., Boonamnuay, S., Kerdprasop, N., Kerdprasop, K.: Neural network-based analysis of precipitation and remotely sensed data. In: Proceedings of the International MultiConference of Engineers and Computer Scientists. vol. 1 (2016)

2. Buzzi, M.A., Rueter, B.L., Ghermandi, L.: Múltiples índices espectrales para predecir la variabilidad de atributos estructurales y funcionales en zonas áridas. Ecología austral 27(1), 55-62 (2017)

3. Carvajal Ramírez, F., Aguilar Torres, M.Á., Agüera Vega, F., Aguilar Torres, F.J.: Clasificación de una imagen multiespectral de satélite de alta resolución espacial mediante redes neuronales artificiales 
4. Chang, J., Clay, D.E., Dalsted, K., Clay, S., O’Neill, M.: Corn (1.) yield prediction using multispectral and multidate reflectance. Agronomy journal 95(6), 1447-1453 (2003)

5. Chen, Y., Lin, Z., Zhao, X., Wang, G., Gu, Y.: Deep learning-based classification of hyperspectral data. IEEE Journal of Selected topics in applied earth observations and remote sensing 7(6), 2094-2107 (2014)

6. Chen, Y., Zhao, X., Jia, X.: Spectral-spatial classification of hyperspectral data based on deep belief network. IEEE Journal of Selected Topics in Applied Earth Observations and Remote Sensing 8(6), 2381-2392 (2015)

7. Evora Jiménez, E.: Sistema de procesamiento de imágenes NIR e IR aéreas para agricultura de precisión. Ph.D. thesis, Universidad Central" Marta Abreu"de Las Villas. Facultad de Ingeniería Eléctrica. Departamento de Automática y Sistemas Computacionales (2016)

8. Foody, G.M.: Thematic mapping from remotely sensed data with neural networks: Mlp, rbf and pnn based approaches. Journal of Geographical Systems 3(3), 217-232 (2001)

9. Galárraga Cañizares, J.L.: Clasificador de hojas mediante Deep Learning. Ph.D. thesis, ETSI_Informatica (2017)

10. Gilabert, M.A., González-Piqueras, J., García-Haro, J.: Acerca de los índices de vegetación. Revista de teledetección 8(10) (1997)

11. Gómez-Casero, M., López-Granados, F., Peña-Barragán, J., Jurado-Expósito, M., García-Torres, L.: Caracterización espectral multitemporal de cultivos de regadío aplicando análisis discriminante y redes neuronales

12. Güemes Ricalde, F.J., Echazarreta González, C., Villanueva, R., Pat Fernández, J.M., Gómez Alvarez, R.: La apicultura en la península de yucatán. actividad de subsistencia en un entorno globalizado. Revista Mexicana del Caribe 8(16) (2003)

13. Li, F., Gnyp, M.L., Jia, L., Miao, Y., Yu, Z., Koppe, W., Bareth, G., Chen, X., Zhang, F.: Estimating $\mathrm{n}$ status of winter wheat using a handheld spectrometer in the north china plain. Field Crops Research 106(1), 77-85 (2008)

14. Lin, Z., Chen, Y., Zhao, X., Wang, G.: Spectral-spatial classification of hyperspectral image using autoencoders. In: Information, Communications and Signal Processing (ICICS) 2013 9th International Conference on. pp. 1-5. IEEE (2013)

15. Lizarazo, I.: Clasificación de la cobertura y del uso del suelo urbano usando imágenes de satélite y algoritmos supervisados de inteligencia artificial. UD y la geomática (2), 4-18 (2008)

16. Maggiori, E., Tarabalka, Y., Charpiat, G., Alliez, P.: Convolutional neural networks for large-scale remote-sensing image classification. IEEE Transactions on Geoscience and Remote Sensing 55(2), 645-657 (2017)

17. Makantasis, K., Karantzalos, K., Doulamis, A., Doulamis, N.: Deep supervised learning for hyperspectral data classification through convolutional neural networks. In: Geoscience and Remote Sensing Symposium (IGARSS), 2015 IEEE International. pp. 4959-4962. IEEE (2015)

18. Mas, J.F.: Un método para combinar datos espectrales e información auxiliar en una red artificial neuronal. Anais XII Simpósio Brasileiro de Sensoriamento Remoto pp. 3543-3549 (2005)

19. Meza, R.R.B., Acuña, J.R.: Clasificación de la salinidad del suelo mediante imágenes de satélite y las redes neuronales artificiales. ECIPeru: Revista del Encuentro Científico Internacional 10(1), 4-8 (2013)

20. Morris, E.P., Gómez Enri, J., et al.: Teledetecion de Habitats Bentonicos en la Bahia de Cadiz, Espana. Ph.D. thesis, Universidad de Cádiz (2011) 
Enfoque para la clasificación de vegetación polinífera usando imágenes multiespectrales ...

21. Paruelo, J.M.: La caracterización funcional de ecosistemas mediante sensores remotos. Revista Ecosistemas 17(3), 4-22 (2008)

22. Paruelo, J.M.: La caracterización funcional de ecosistemas mediante sensores remotos. Revista Ecosistemas 17(3) (2008)

23. Ponce Suarez, C.Y.: Análisis multitemporal de la cobertura vegetal del valle interandino del chota e identificación de zonas de restauración ecológica. B.S. thesis (2017)

24. Schneider, G., Hadad, A.J., Kemerer, A.: Implementación de un software para el análisis de imágenes aéreas multiespectrales de caña de azúcar [implementation of software for the analysis of multispectral aerial images sugarcane]. Ventana Informática (28) (2013)

25. Suarez, A., Jiménez, A., Franco, M.C., Cruz-Roa, A.: Clasificación automática de coberturas del suelo en imágenes satelitales utilizando redes neuronales convolucionales: Un caso aplicado en parques nacionales naturales de colombia

26. Tovar, S.A.O., Valenzuela, D.M.Q., Ortega, Y.D.M., Pastrana, D.A.M., Medina, W.A.O., Portilla, D.E.P.: Zonificación de unidades productivas con el uso de herramientas satelitales y actividades de formación interdisciplinar. Revista del Sistema de Ciencia Tecnología e Innovación (SENNOVA) 2(1), 98-121 (2017)

27. Vincini, M., Frazzi, E., D'Alessio, P.: A broad-band leaf chlorophyll vegetation index at the canopy scale. Precision Agriculture 9(5), 303-319 (2008) 